\title{
New insight on old-timers
}

Memory T cells ensure that the immune response is more effective against re-infecting pathogens. Two key distinctions between memory $\mathrm{CD}^{+} \mathrm{T}$ cells and their naive counterparts are crucial for this: first, memory $\mathrm{T}$ cells have distinct migratory patterns; and second, they survive for longer. New studies by Steinert et al. and Cui et al. offer fresh insight into both of these aspects of memory.

Memory $\mathrm{CD}^{+} \mathrm{T}$ cells have been divided into distinct subsets on the basis of putative trafficking and functional properties. Central memory $\mathrm{T}$ $\left(\mathrm{T}_{\mathrm{CM}}\right)$ cells are suggested to be longlived memory cells that recirculate via secondary lymphoid organs (SLOs), whereas effector memory $\mathrm{T}$ $\left(\mathrm{T}_{\mathrm{EM}}\right)$ cells are thought to primarily

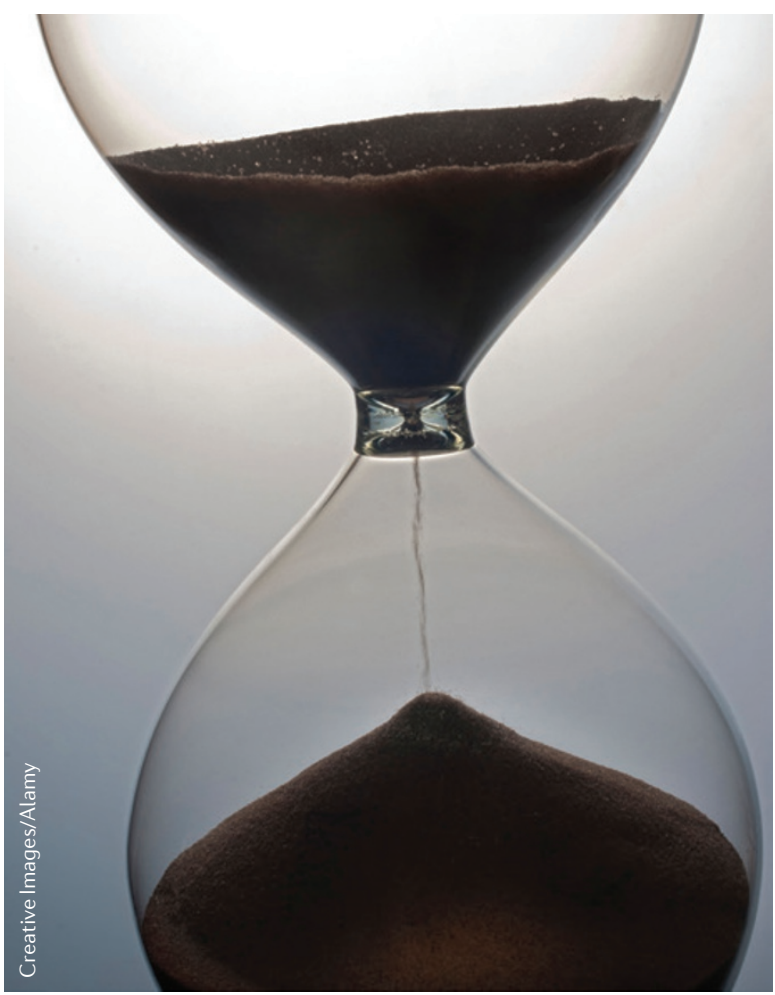

recirculate between the blood and non-lymphoid tissues and respond rapidly to re-infecting pathogens. A further set of tissue-resident memory $\mathrm{T}\left(\mathrm{T}_{\mathrm{RM}}\right)$ cells have been described that are retained in non-lymphoid tissues and do not recirculate. The relative contribution of each subset to immune memory has been unclear; to address this, Steinert et al. developed a quantitative immunofluorescence microscopy (QIM) method. In a mouse model of lymphocytic choriomeningitis virus (LCMV) infection, standard protocols for cell isolation markedly underestimated the frequencies of LCMV-specific memory CD8 ${ }^{+} \mathrm{T}$ cells in tissues, particularly in non-lymphoid sites, when compared with QIM. Indeed, QIM analyses suggested that more memory $\mathrm{CD}^{+} \mathrm{T}$ cells are found in the blood and peripheral tissues than in SLOs.

Parabiosis experiments showed that most memory $\mathrm{CD} 8^{+} \mathrm{T}$ cells in peripheral tissues do not recirculate. This suggests that the majority of memory $\mathrm{CD}^{+} \mathrm{T}$ cells in nonlymphoid tissues are $\mathrm{T}_{\mathrm{RM}}$ cells, rather than $\mathrm{T}_{\mathrm{EM}}$ cells, although bona fide $\mathrm{T}_{\mathrm{EM}}$ cells could be detected exiting tissues via lymphatics. CD69 expression is often used to define $\mathrm{T}_{\mathrm{RM}}$ cells, but the authors found that a substantial number of resident memory $\mathrm{CD}^{+} \mathrm{T}$ cells do not express this marker. Furthermore, many of the memory $\mathrm{CD}^{+} \mathrm{T}$ cells that entered peripheral tissues (a migratory behaviour associated with $\mathrm{T}_{\mathrm{RM}}$ cells or $\mathrm{T}_{\mathrm{EM}}$ cells) expressed the lymph node-homing molecule CD62L, which is typically used to define $\mathrm{T}_{\mathrm{CM}}$ cells. In fact, adoptive transfer experiments showed that purified $\mathrm{T}_{\mathrm{CM}}$ cells and $\mathrm{T}_{\mathrm{EM}}$ cells were equally efficient at migrating to inflamed peripheral tissues. These data suggest that tissue-resident memory $\mathrm{CD}^{+}$ T cells markedly outnumber those that are recirculating and that our current paradigms of memory $\mathrm{T}$ cell subsets require revision.

Cui et al. explored how metabolic processes regulate longevity in memory $\mathrm{CD} 8^{+} \mathrm{T}$ cells. They compared gene expression profiles of naive, effector and memory CD8 ${ }^{+} \mathrm{T}$ cells and found that the glycerol channel aquaporin 9 (AQP9) was selectively expressed by memory $\mathrm{CD} 8^{+} \mathrm{T}$ cells. These cells were shown to upregulate AQP9 in response to stimulation with interleukin-7 (IL-7) — and, to a lesser extent, in response to IL-15 and deficiency of AQP9 impaired the survival of memory but not effector $\mathrm{CD}^{+}{ }^{+} \mathrm{T}$ cells during LCMV infection.

Further experiments suggested that AQP9 deficiency impairs memory $\mathrm{CD}^{+} \mathrm{T}$ cell survival by preventing glycerol uptake, which is required for the synthesis of triglycerides. Triglycerides serve as a source of fatty acids for fatty acid oxidation, a metabolic process that generates energy for memory T cell survival. Consistent with this, AQP9-deficient memory CD8 ${ }^{+} \mathrm{T}$ cells had reduced ATP levels, and the overexpression of genes involved in triglyceride synthesis prolonged their survival. Additional analyses showed that IL-7 also increases the expression of genes involved in triglyceride synthesis in previously activated but not naive $\mathrm{CD}^{+} \mathrm{T}$ cells. Therefore, IL-7 promotes memory CD $8^{+} \mathrm{T}$ cell survival not only through the induction of anti-apoptotic genes but also by supporting their metabolic activities.

Yvonne Bordon

ORIGINAL RESEARCH PAPERS Steinert, E. M. et al.

Quantifying memory CD8 T cells reveals regionalization of immunosurveillance. Cell 161, 737-749 (2015) | Cui, G. et al. IL-7-induced glycerol transport and TAG synthesis promotes memory CD8 ${ }^{+} T$ cell longevity. Cell 161, 750-761 (2015) 